\title{
Efficient simulation of mechanism kinematics using bond graphs
}

\author{
G. Romero , J. Felez, J. Maroto, J.M. Mera \\ ETS Ingenieros Industriales, Universidad Politécnica de Madrid (UPM), José Gutierrez Abascal, 2, 28006 Madrid, Spain
}

Keywords:

Bond-Graph

Differential-algebraic equations

Dynamics

Kinematics

Mechanism

Earth moving machines

\begin{abstract}
A B S T R A C T
This paper presents a methodology for obtaining the equations corresponding to a mechanism that are necessary for carrying out a kinematic simulation. A simulation of this kind means obtaining the coordinates dependent on the system according to the movements imposed by the degrees of freedom. Unlike a dynamic simulation, where the set of elements moves according to the different external forces existing, in kinematic simulation the movement of the whole set depends exclusively on imposing movement on one or more of the bodies according to the degrees of freedom initially possessed by the mechanism.

After presenting an analysis of how to obtain the necessary equations for several simple systems, this methodology is applied to the particular case of a front-loader, where in order to move and tilt the bucket, various closed mechanisms are integrated.
\end{abstract}

\section{Introduction}

Real time simulation is an essential requirement in simulations such as those of vehicle dynamics, where the driver expects an immediate response, as is the case in a real situation. The implementation of each and every part of a vehicle, together with the fact that they are more and more complicated, means that this real time simulation requires a thorough in-depth analysis aimed at simplifying to the maximum everything that is either not strictly essential or requires more information than necessary.

When it comes to simulating machinery such as backhoes, wheel loaders, there are two parts that come together; that corresponding to the dynamics of the vehicle itself, and the part relative to the movement of implements such as bucket, arms, actuators, etc.

The aim of this article is to demonstrate the validity of implementing the relevant kinematic equations corresponding to the bucket movement mechanism instead of dynamic equations. Thus, the set of equations needed to obtain the movement of the whole assembly will depend solely on the movement of the main arms instead of on the external actions existing in all of the implements.

In recent years, the generation of dynamic equations of systems modelled with bond graphs has been the topic of considerable research. These equations have been presented in a variety of forms [1]. Classical formulations express these equations in terms of a large number of momentum associated to inertances and displacements associated to compliances. These elements can present integral or differential causality. In order to obtain these equations, it is necessary to establish the causality in the model. Karnopp and Margolis [2] contributed with the stiff compliance approach, where high stiffness compliances are introduced in the model to eliminate the causal loops between integral and derivative causal storage ports. In this 
case, a set of differential equations is obtained, including as variables the flows associated to the previous inertances, displacements associated to the previous compliances, and the corresponding variables associated to the new stiff elements introduced, considerably increasing the number of differential equations to be solved. Usually, this approach needs the use of special numerical solvers for stiff differential equations, requiring very small integration step times. For these reasons, this procedure is not appropriate for simulation in real time.

Another approach was the introduction of Lagrange multipliers [3] in the model. Some other authors [4] introduce residual sinks and sources $[5,6]$ in a similar solution to the Lagrange approach [7]. When Lagrange multipliers are used to break causal paths, a set of differential-algebraic equations is obtained, composed by a number of differential equations equal to the number of inertances plus compliances, and a number of algebraic (or constraint) equations equal to the number of Lagrange multipliers. The number of equations is the same as with the previous method, this method being also not appropriate for real time. Nevertheless, this approach presents important formulation advantages that will be considered in this work.

Previous methods prevent the existence of causal loops, but it is necessary to pre-analyse the model and to modify the model in a subsequent operation [8]. Another approach to solve the problem is the use of break variables [9,10] to open the causal loops. Causal loops always present an algebraic character. Algebraic loops relate their internal variables by means of algebraic relationships. It means that these kinds of loops do not involve integration operations. This fact leads to the definition of Zero-order Causal Paths ZCPs [11]. The mathematical model obtained from bond graphs with ZCPs and opened with break variables is also a differential-algebraic equation set (DAE) [12].

This last approach reduces the number of equations to a number equal to the number of inertances plus compliances plus break variables, but it is not enough for real time.

The use of Lagrange multipliers or the introduction of break variables implies the necessity of new variables and the appearance of constraint equations, increasing considerably the number of involved variables and the number of equations to be solved. These procedures are very useful to obtain the system equations in a systematic way, but they have to be improved or modified for their use in real time simulation.

\section{Front-loader description}

Front-loaders are powerful machines with a large load capacity that are specially designed for light excavation work with smooth or previously turned over material, earth moving tasks or extraction and sand and cement collection in gravel pits and asphalt manufacturing plants.

There are basically two kinds, depending on their type of drive, either with caterpillar tracks or tyres. While a caterpillar track design makes them ideal for terrain that is rugged or of difficult access, wheeled vehicles are suitable for smooth, flat or slightly sloping surfaces. This last type has a central articulation between its front and rear part to give it greater efficiency of movement, this being the vehicle on which the study will be focussed.

In addition, such vehicles are fitted with a bucket mounted on articulated arms fixed to the front part of the chassis and operated by hydraulic mechanisms.

The machine can be broken down into two clearly differentiated parts (Fig. 1). The first part is the chassis, the steering and the wheels of the vehicle together with its different engine and transmission assemblies. The second part contains all that is relative to bucket movement including the different arms and hydraulic actuators; it is this part that will be studied in this paper.

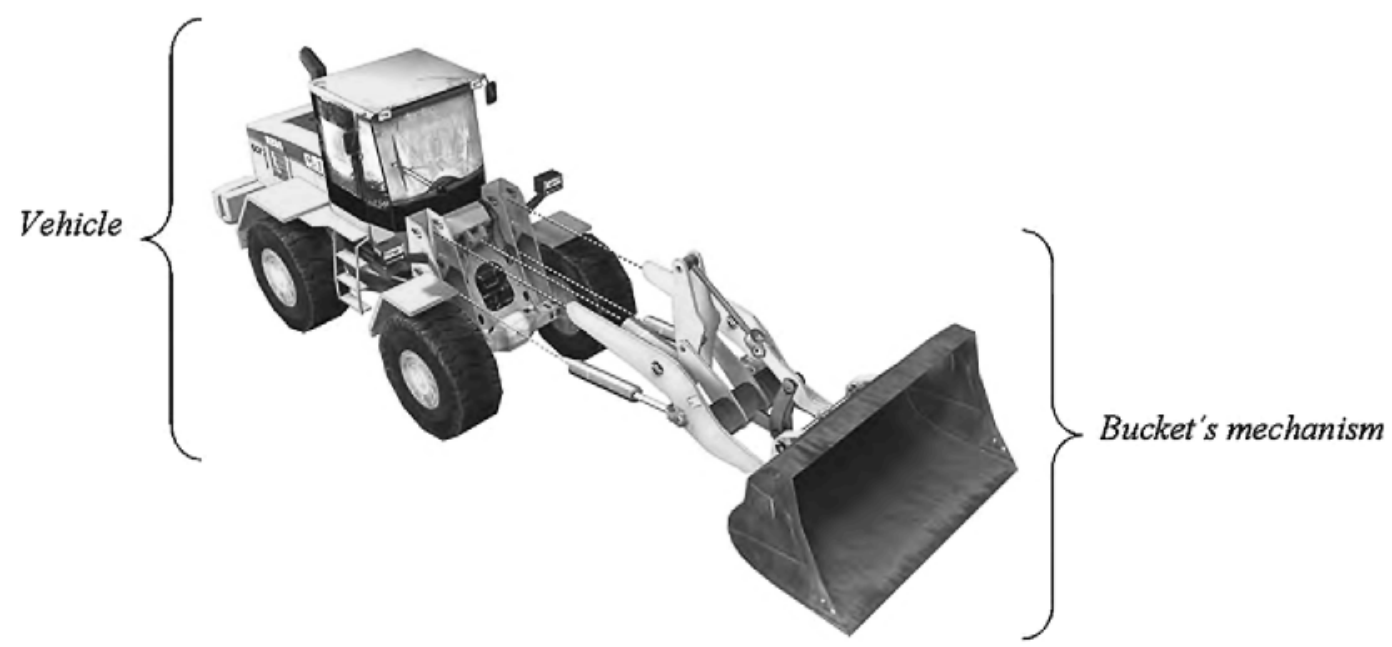

Fig. 1. Parts of a front-loader. 


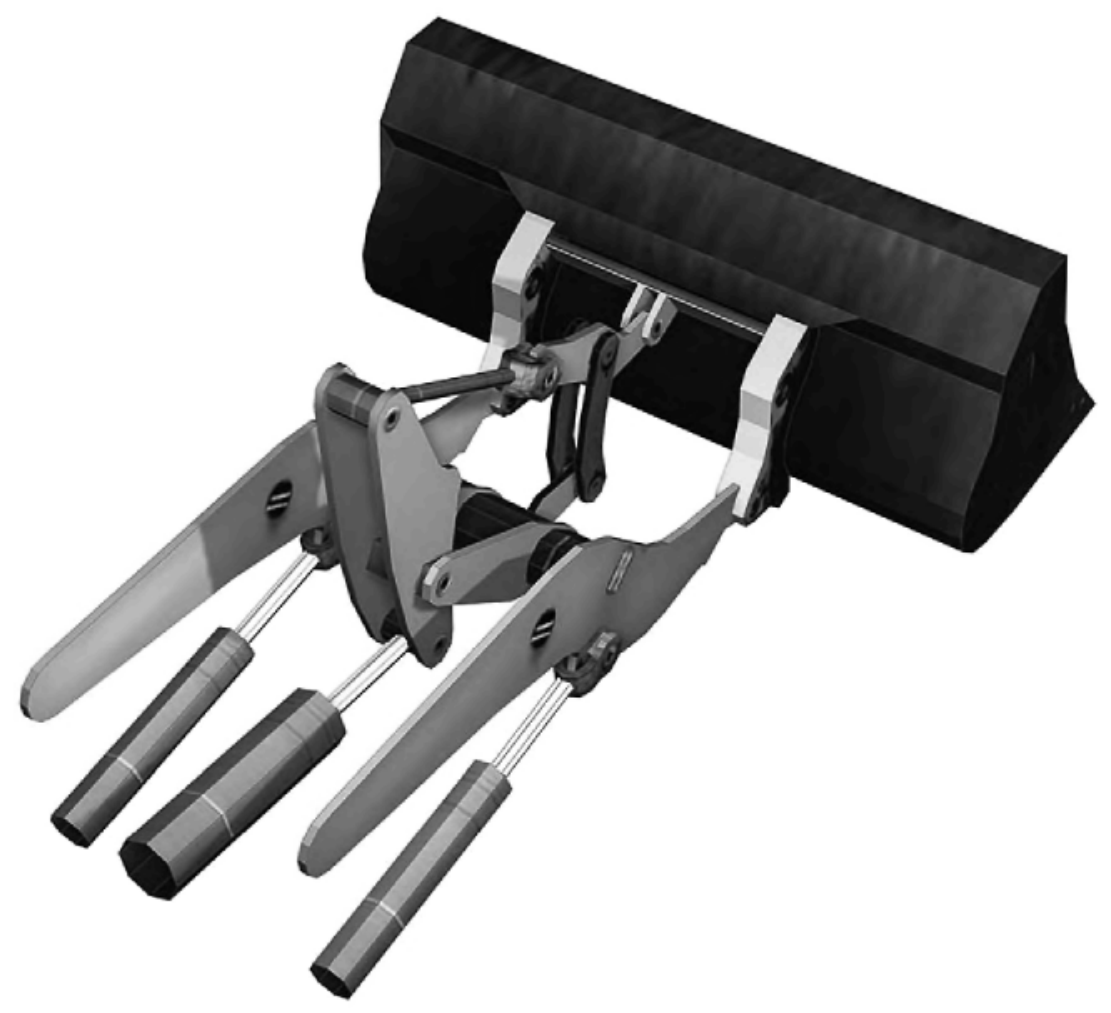

Fig. 2. Bucket components.

By using the different arm elements (Fig. 2), bucket movement can be achieved, thanks to which the movements for loading and unloading materials can performed. This part is made up of the arms, struts, bucket and actuators.

The concept of the degrees of freedom may be defined as the number of entries that need to be provided in order to give rise to a predictable exit. Each type of entry required will need some kind of a starter or an actuator, either in the form of a solenoid engine or a hydraulic cylinder. The mechanism has two degrees of freedom and the movement of the different parts is produced by operating the three actuators, the ones at either side working simultaneously. The action of the side actuators responds to one of the levers in the cab while the central actuator responds to another, both being handled by the operator.

As this mechanism is fixed to the front part of the machine, the reactions existing on the machine need to be inserted as external forces and torques due to the interaction of the bucket with the ground and the load.

It can be seen how the mechanism is made up of a series of elements located in a parallel form on both sides and of others located on the plane of symmetry. The first ones serve to raise or lower the bucket, while the second ones are used to tilt it.

However, it may be assumed that the load contained in the bucket is uniformly distributed.

Since both mechanisms work on a single plane, that is, the $X Z$ plane corresponding to the front chassis, a plane model (Fig. 3) can be used instead of a more complex three-dimensional one. Thus, by superimposing the two mechanisms on a single projection plane, the final mechanism to be simulated is obtained.

\section{Reference coordinates}

As we know, kinematics studies the movements of particles and stiff bodies without taking account of the forces needed to give rise to these movements. Statics is the study of mechanical systems where the resultant of the system of forces is zero, and is thus balanced (at rest or moving at constant velocity). Finally, when this resultant is not zero, the mechanical system is charged with accelerated movement, and these unbalanced forces and the movements they give rise to comprise the field of study called dynamics.

When it comes to studying a mechanism's movement, it is essential to choose one reference system or another. Thus, a system of local coordinates will usually be chosen when studying three-dimensional solids, which requires a change of reference systems in the existing joints of a mechanism of this type. When analysing the behaviour of a mechanism that only moves on a plane, it turns out to be much simpler to work with a system of coordinates that are parallel to the global system (Fig. 4), which avoids having to change the reference systems in the joints. This is why a representation of a flat rod using a Bond-Graph (Fig. 5) will consist of three inertial type ports $\left(I_{x}, I_{y}\right.$ and $\left.I_{f}\right)$. 


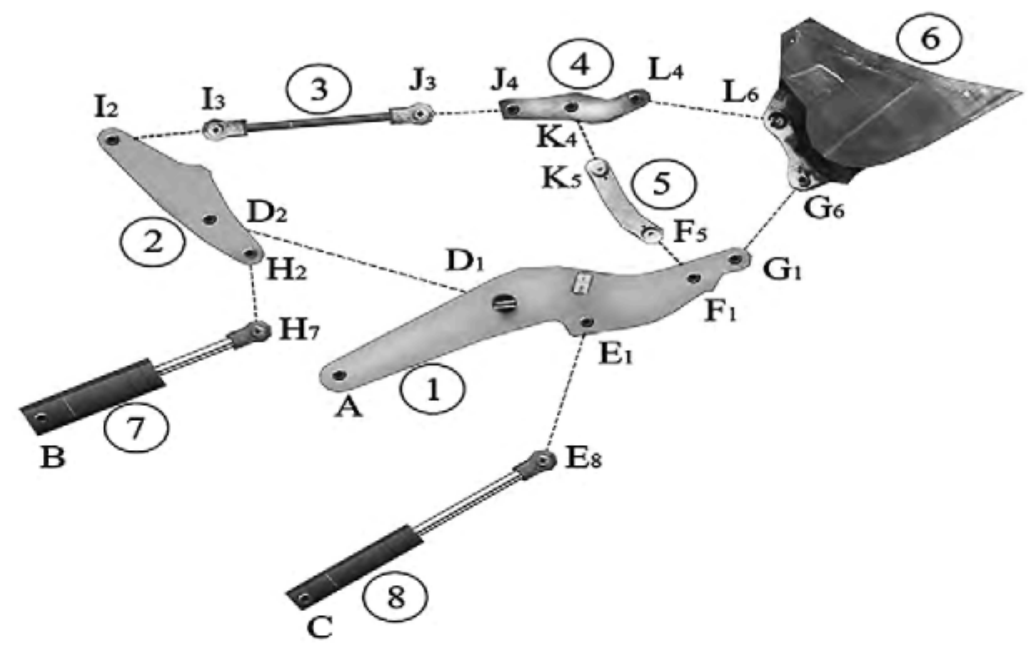

Fig. 3. Plane loading mechanism.

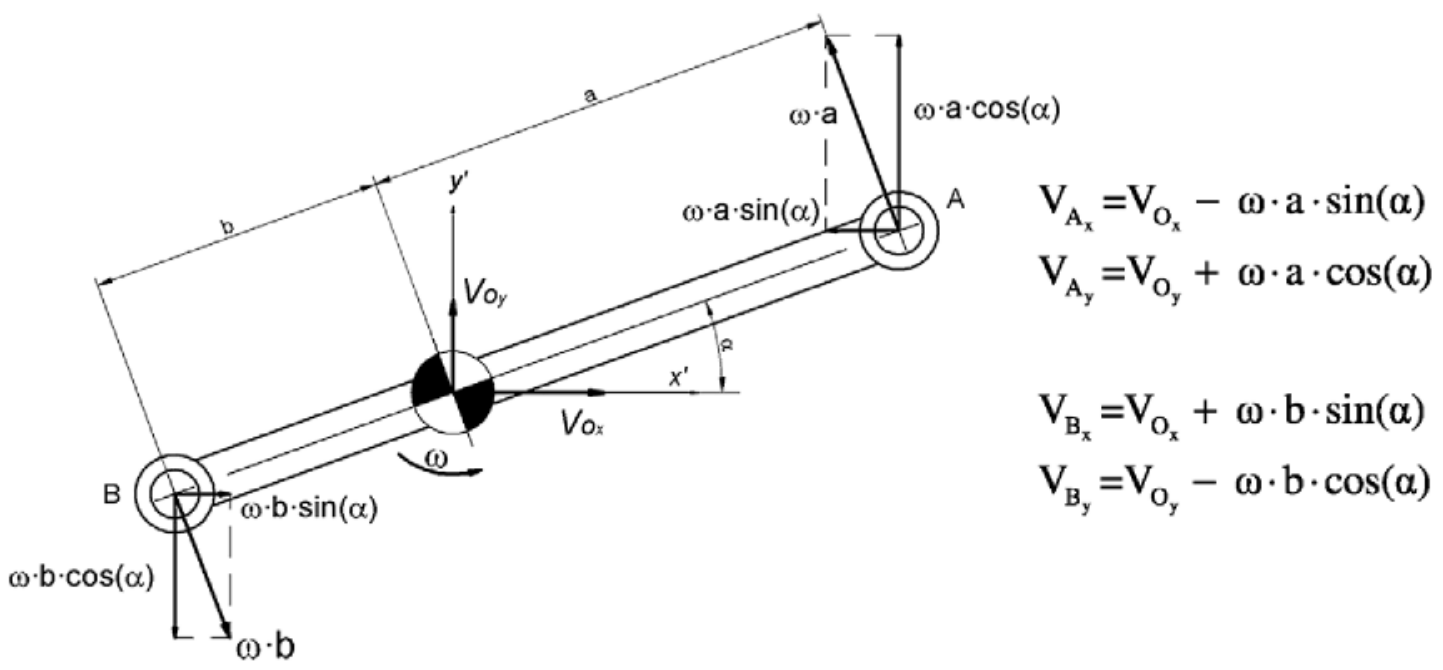

Fig. 4. Planar 2D rod in global coordinates.

In this figure, we can see the name assigned to the element $(T F 2, I X, \ldots)$, two dots (:) and the expression associated with it $(-b \cdot \cos (X I J), M \ldots$, respectively). The variables associated with the different ports are $P(m \cdot V)$ and $X$ in the case of Inertances and Compliances, respectively. If we write $V_{t 1}$, we think of the velocity of an Inertance called 'I1', but in the case of the mechanism we need to introduce the integral of $V_{I 1}$ into the MTF elements, calling it the angular displacement $\left(\alpha=X_{I J}=f\left(V_{I J}\right) \mathrm{d} t\right)$. For this reason in the next pages I will use this notation to represent displacements $\left(X_{\text {Name }}\right)$ and velocities $\left(V_{\text {Name }}\right)$.

To obtain the equations for the bucket movement mechanism, each of the parts will have to be studied in isolation, and then they will be assembled according to the restriction equations. To do this, the coordinates in respect of a global reference system for each of the points existing in the different bodies and which serve to join them all together can be obtained using the centre of gravity coordinates $X_{i}$ and $Y_{i}$ and the product of the transformation matrix depending on the angle $\varphi_{i}$ and the matrix with the coordinates of the different points in a local reference system.

\section{Hydraulic actuators}

These are the components whose job is to move the bucket by means of the thrust arm and the unloading lever (Fig. 6). Their lengths at any instant are $L_{\mathrm{cil} 1}$ and $L_{\mathrm{cil} 2}$, respectively. The effect of these cylinders is twofold as hydraulic force is used both to push the bucket and to contract it. 


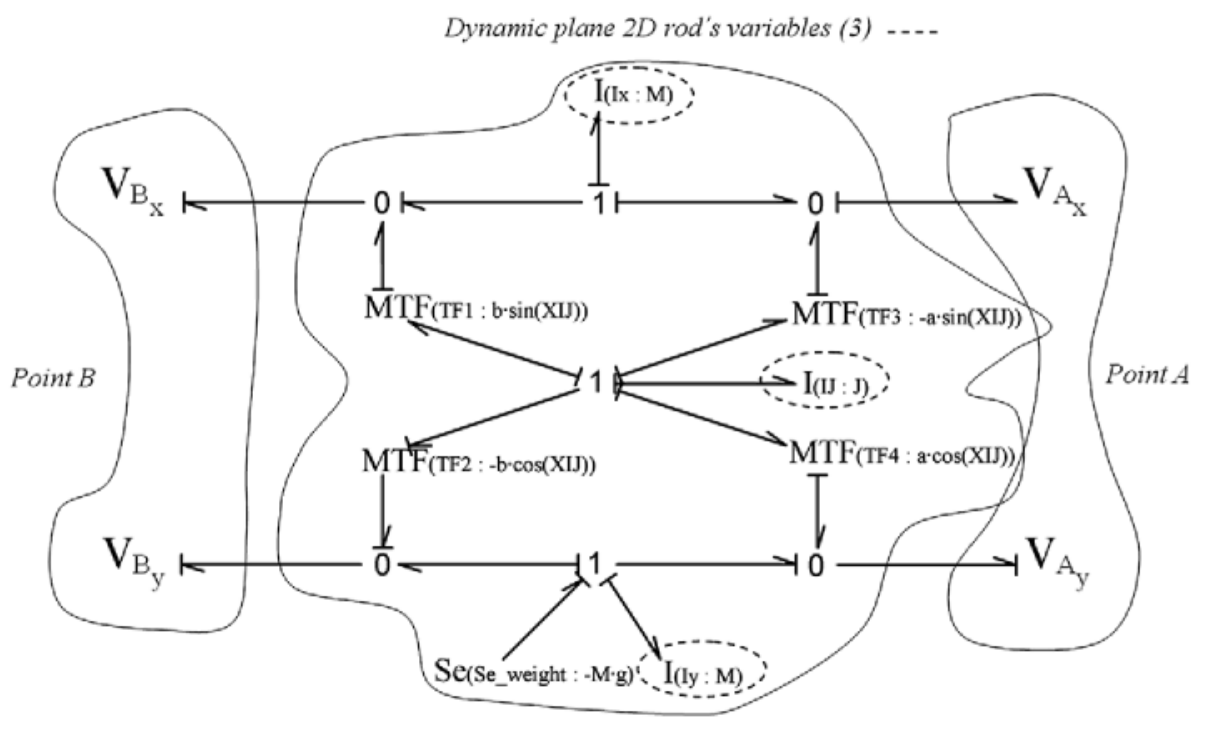

Fig. 5. Plane 2D rod in global coordinates using a Bond-Graph.

The coordinates of the points corresponding to the end of the hydraulic cylinders can be obtained in the same way as for the other bodies by means of its length $L_{\text {cil1 }}$ or $L_{\text {cil2 }}$, the centre of gravity coordinates $X_{i}$ and $Y_{i}$ and the angle $\varphi_{i}$.

In order to be able to simulate the movement corresponding to the arm-bucket mechanism using the controls, instead of associating the equations of the hydraulic circuit that acts on the support arm and unloading cylinders, we can operate directly on the increase in the length of cylinders $L_{\text {cil1 }}$ and $L_{\text {cil2. }}$. To do this, it is sufficient to take account of the position of the controls that handle each of the actuators, the load weight and the engine speed. It is essential to do things in this way, since if all the elements in the intervening period are inserted the simulation will be unnecessarily slowed down.

In order to see how the cylinders perform in accordance with the parameters commented on in the previous paragraph, the different valves were reproduced (distributors, anti-return, pressure limiters, etc.), pumps, hydraulic cylinders, and other parts to be found in the hydraulic circuit of an actual machine and then an isolated simulation was carried out to see what happens in the said machine (Fig. 7).

To see what would happen by operating the two controls at the same time, initially the upstroke was simulated in the two actuators and then the downstroke.

For a constant work regime of the pump, which is the equivalent of working at a constant revolution speed with each of the controls being in a fixed position, it can be seen (Fig. 7) that the speed of the cylinders is less if they are both working at the same time, it increasing slightly as one of them reaches the limit. Indeed, since the pump propels a constant volume this needs to be shared between the circuits of each of the actuators, the whole flow entering one of them when the other has reached its limit (Fig. 7).

However, it can also be seen that for a relatively small load the speed with which one of the actuators moves is greater than in the case of a greater load, just as would be expected, since the pump, at a constant regime, always exerts the same pressure.

If the engine speed in the machine is increased, the pump will increase in the same proportion, causing an increase in the speed at which the actuators move, similar to what occurs if an increase in the position of the controls is produced.

Therefore, the increase in the length of the cylinders $L_{\mathrm{cil1}}$ and $L_{\mathrm{cil2} 2}$ will be produced at a speed proportional to the engine revolution speed and at the position of the corresponding control, it being negatively influenced on increasing the load or when the control of the other actuator is operated in parallel. This will be particularly useful, since in this way we avoid having to calculate both the forces exerted by the corresponding actuators and the stabilisation of their values, in favour of conducting a prior experiment from which some range of working speeds of valid displacements can be obtained.

If an actuator of this type is to be simulated, it must be borne in mind that it resides in its own local coordinate system, that one of its ends constantly changes its position with respect to a fixed point (Fig. 8), and that it does so in accordance with a speed we have assumed to be dependent on the status of the control levers and the load in the bucket.

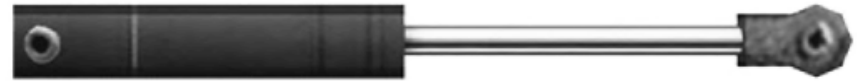




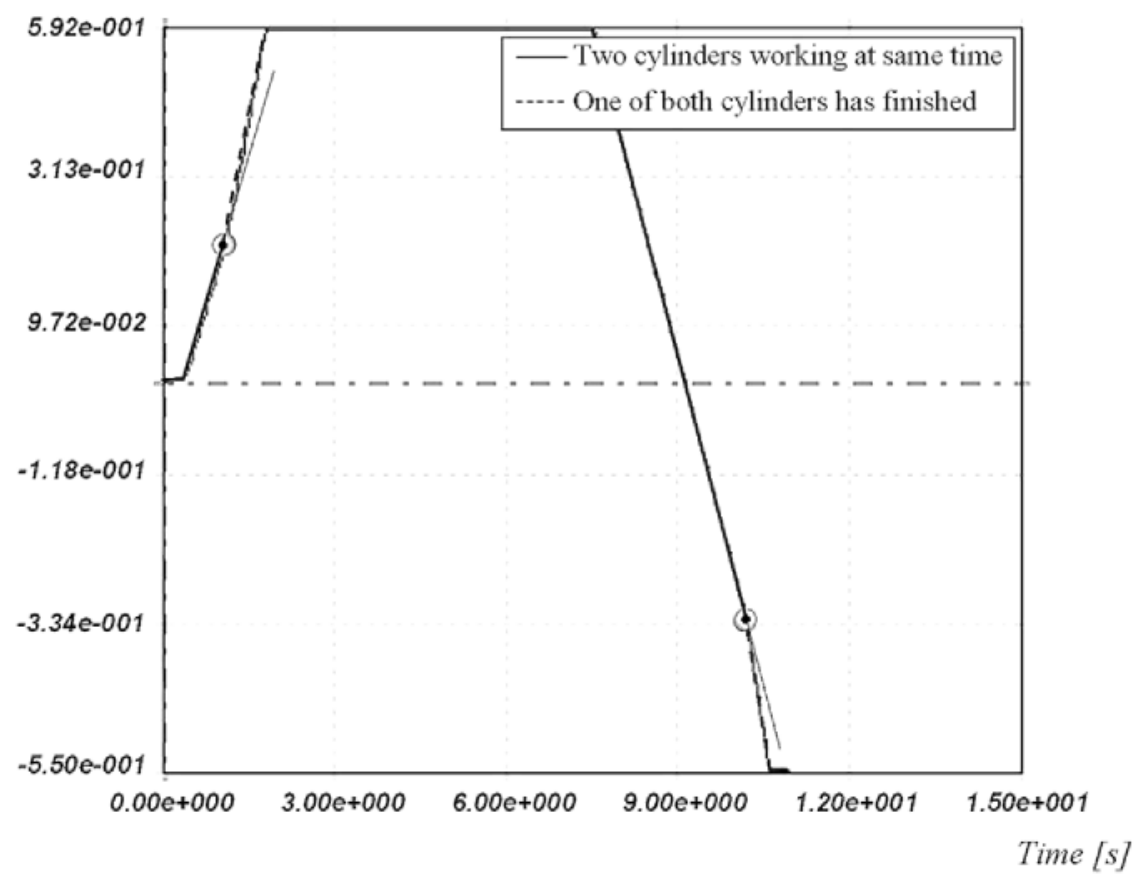

Fig. 7. Movement of the support arm cylinder.

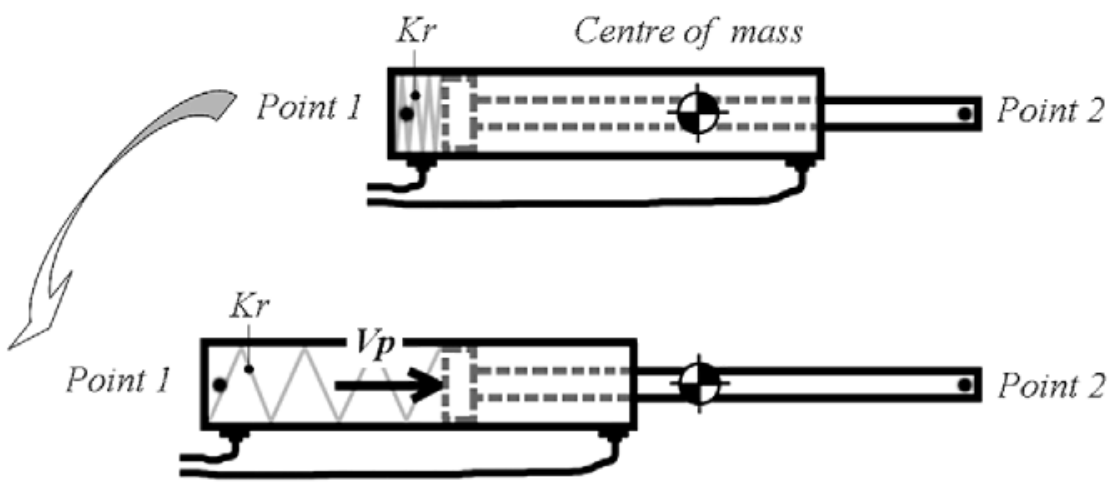

Fig. 8. Hydraulic actuator.

Taking its operating characteristics into account, a hydraulic actuator could be modelled using Bond-Graph notation as follows (Fig. 9):

As shown in Fig. 9, the actuator consists of a plane bar in local coordinates, represented by the 3 inertias Ipiston_x, p Ipiston $\_y$ and Ipiston_z and element GY. The two endpoints are related to the angular velocity by means of elements TF9 and TF10. In $X_{\text {local }}$ coordinates, while the point located at the right remains at a fixed distance of $+{ }^{L} / 2$ from the centre of gravity, the other point located at the left is at a negative distance of $L / 2$ plus a term that corresponds to the displacement of the piston with respect to its initial position $\left(-\left({ }^{L} / 2\right)+X_{K r}\right)$. In order to know how much the piston is displaced in $X_{\text {local }}$ coordinates, a spring $(K r)$, of zero stiffness, needs to be placed in the direction of its displacement, i.e. near the inertia that represents the movement of the actuator in $X_{\text {local }}$ coordinates.

Therefore, as the piston is gradually displaced at a velocity of $(V p)$ depending on the position of the control levers and the load in the bucket, the spring obtains its displacement and adds it to the initial distance of the moving end, thereby making it possible to know the position of the endpoint at every instant.

Once the position and velocities of the piston have been obtained, a change of reference with respect to the global system must be made for its subsequent assembly using TF1 to TF4 and TF5 to TF8 elements. 


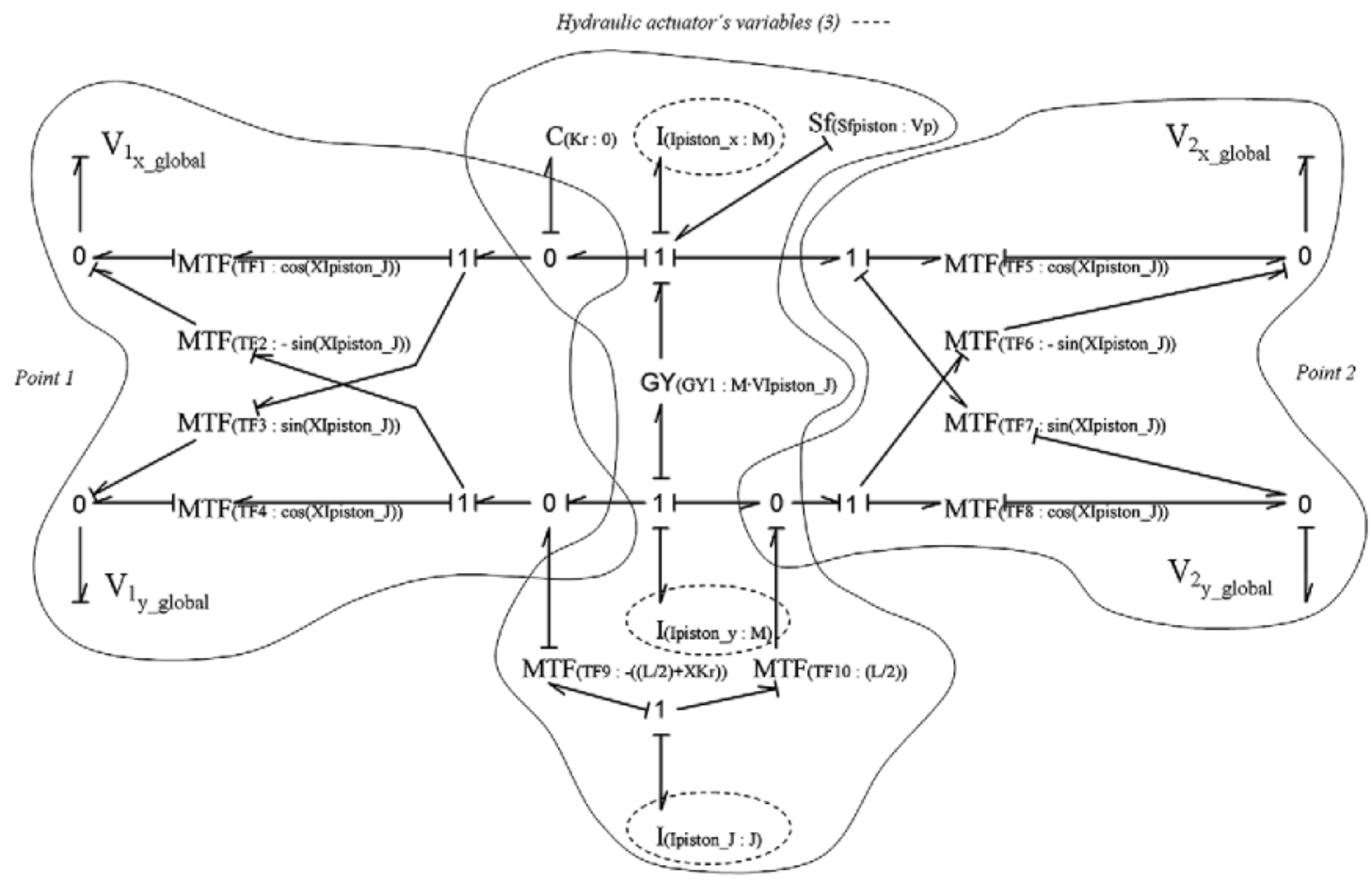

Fig. 9. Hydraulic actuator using a Bond-Graph.

If we assume that the velocity of the actuators is proportional to the position of the control levers and the load in the bucket (5), this means that the forces required to originate these movements need not be taken into account.

$V_{p}=$ function(controllevers, pump, limits, load)

Thus, it may be stated that when dealing with a dynamic simulation where there are as many degrees of freedom initially as impositions of movement, it is possible to eliminate the inertial ports from the model along with the external forces.

However, if the same number of equations is to be formulated, they must be substituted by zero value compliancetype ports, obtaining a model similar to that shown in Fig. 9. We need add a zero value port to the model because we need some variables in the model (angle produced by the two rods) and we cannot delete all the ports.

In this way, we reduce the number of equations to be simulated by formulating only those that correspond to the angular velocities rather than also simulating those corresponding to linear velocities. This change with respect to the traditional flat bar only reduces the number of equations but does not eliminate any problems of causality that may exist in a mechanism such as algebraic or closed loops.

Since the position of all the elements of the mechanism can be obtained by simply knowing the angles of each of the rods, the ports corresponding to the horizontal and vertical displacements can also be eliminated, thereby passing from an initial 3 algebraic equations to 1 equation.

Thus, the rod to be used in mechanisms having as many impositions of velocity as the initial degrees of freedom will contain only one zero value spring-type port (Fig. 10) Ipiston_J in the place of the three inertias.

\section{Plane bar equivalent}

As was seen in Sections 2 and 3, each element can be substituted for a bar-type plane element, the system of coordinates to be used being one parallel to the global system.

As we have stated previously, it may be assumed that the velocity of the actuators is proportional to the position of the control levers and the load in the bucket; we can therefore study the movement of the mechanism as a whole in a kinematic way, without taking the masses and inertias of the system into account. It will only be necessary to decrease the velocity of the actuators as the load supported by the bucket increases.

Therefore, in the same way as was done with the model corresponding to the hydraulic actuator, the plane bar in global coordinates that will be used is the one shown in Fig. 11, where all the inertias are replaced by one zero value spring $(I J)$ [13]. This spring is inserted into the node where there is angular velocity, with the object of obtaining the angle of the bar and 


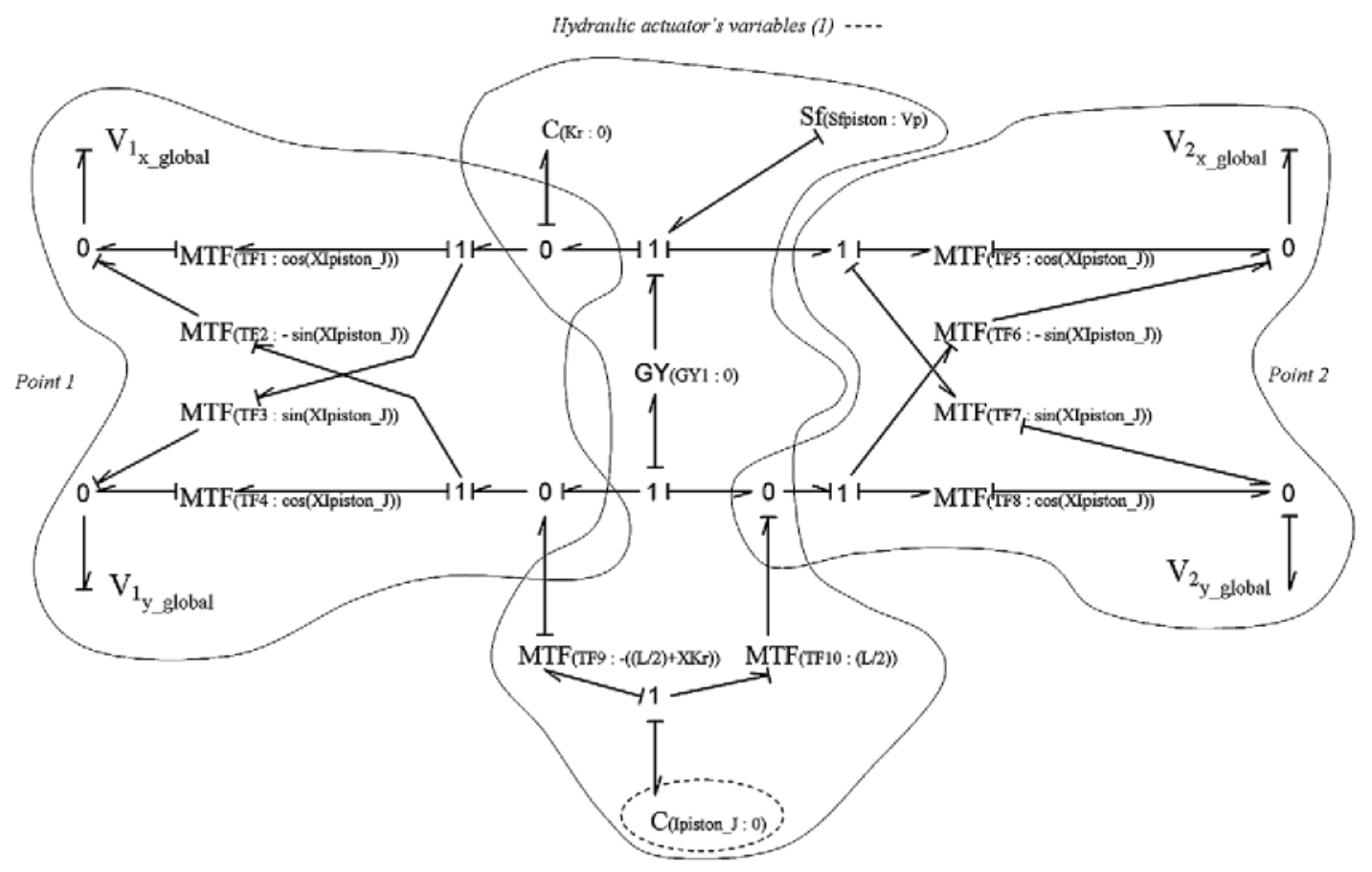

Fig. 10. Hydraulic actuator equivalent using a Bond-Graph.

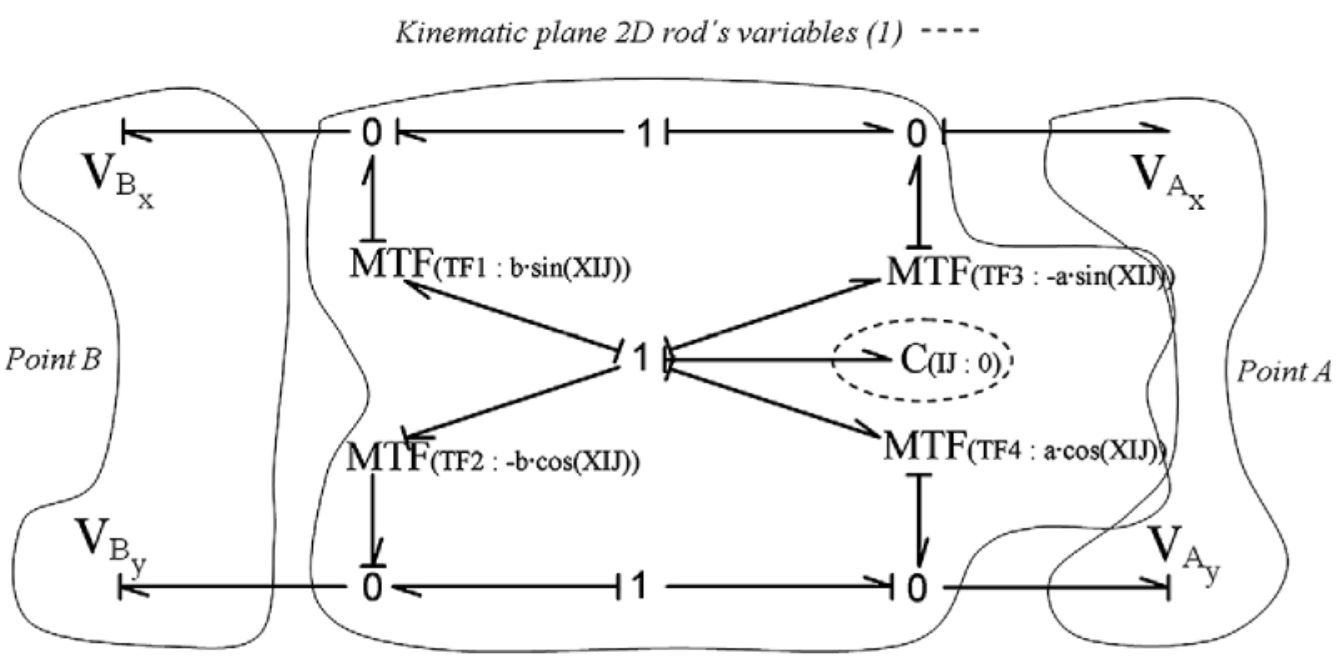

Fig. 11. Plane bar equivalent using a Bond-Graph.

being able to work with it at any moment. The angle of the bar is the spring state and it is equivalent to use a block that integrates the velocity signal from the 1 -junction.

In this way, we reduce the number of equations to be simulated by formulating only those that correspond to the angular velocities rather than also simulating those corresponding to linear velocities. This change with respect to the traditional flat bar only reduces the number of equations but does not eliminate any problems of causality that may exist in a mechanism such as algebraic or closed loops.

\subsection{Simple plane mechanism}

In the following example, a very simple model has been made consisting of a bar and an actuator, as shown in Fig. 12, in order to demonstrate the validity of the models presented here. 


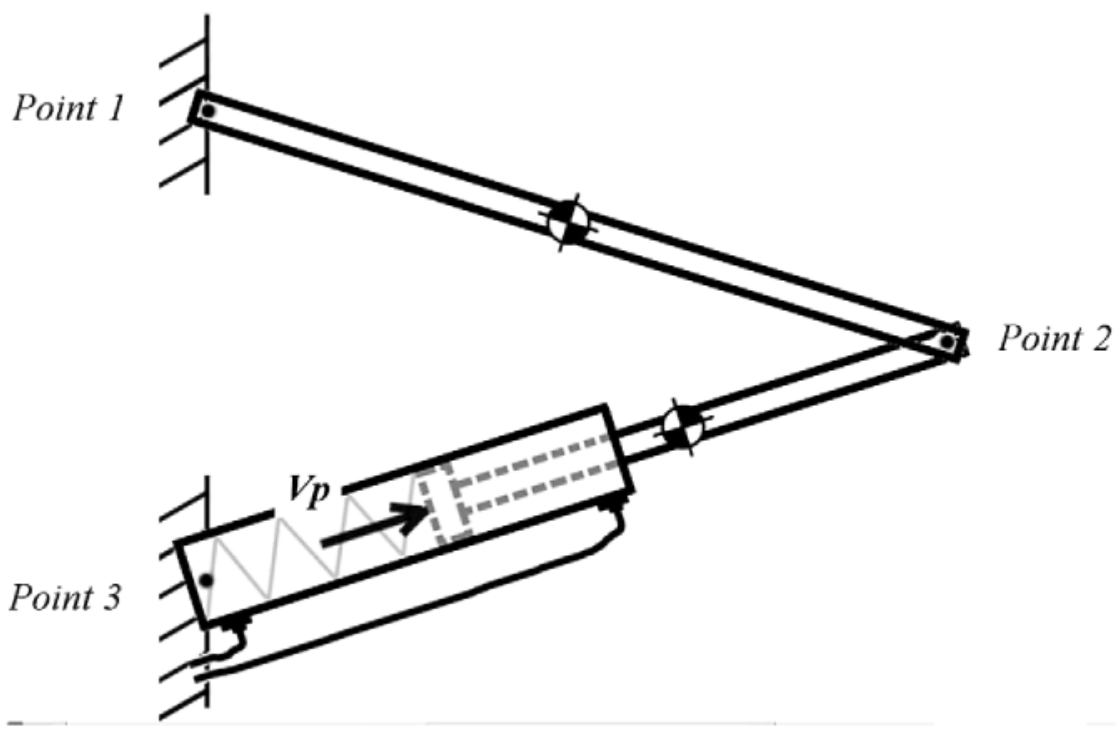

Fig. 12. Hydraulic actuator and plane bar mechanism.

To model this first mechanism, it is sufficient to link the hydraulic and plane bar actuator equivalent models shown in Figs. 10 and 11 using type 1 junctions (Fig. 13).

Once the Bond-Graph model has been completed and the causality resolved, the corresponding equations can be obtained. It may be seen how there are only the three differential equations belonging to each of the springs inserted.

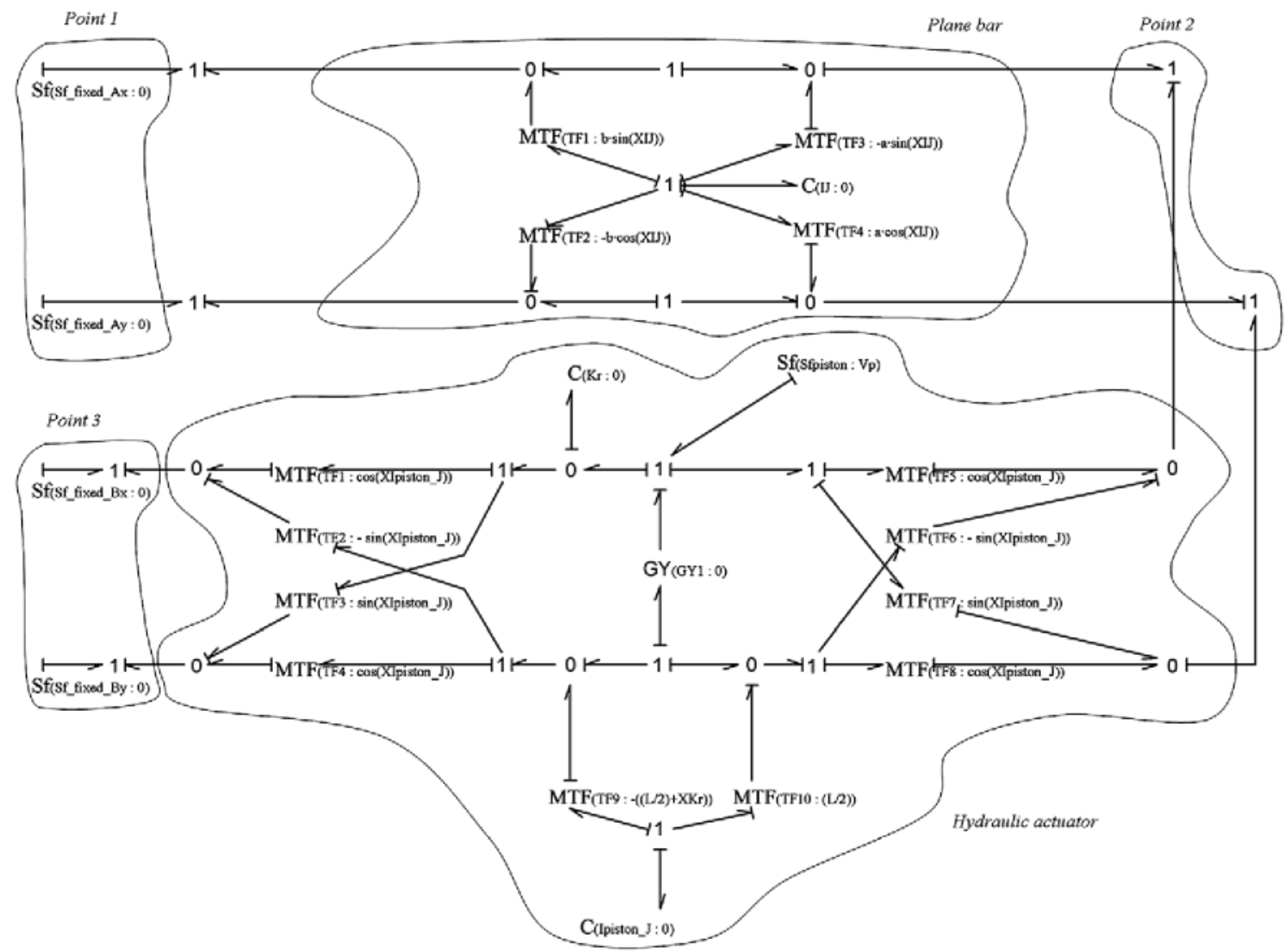

Fig. 13. Hydraulic actuator and plane bar equivalent mechanism using a Bond-Graph. 
Velocity of the hydraulic actuator $[\mathrm{m} / \mathrm{s}]$

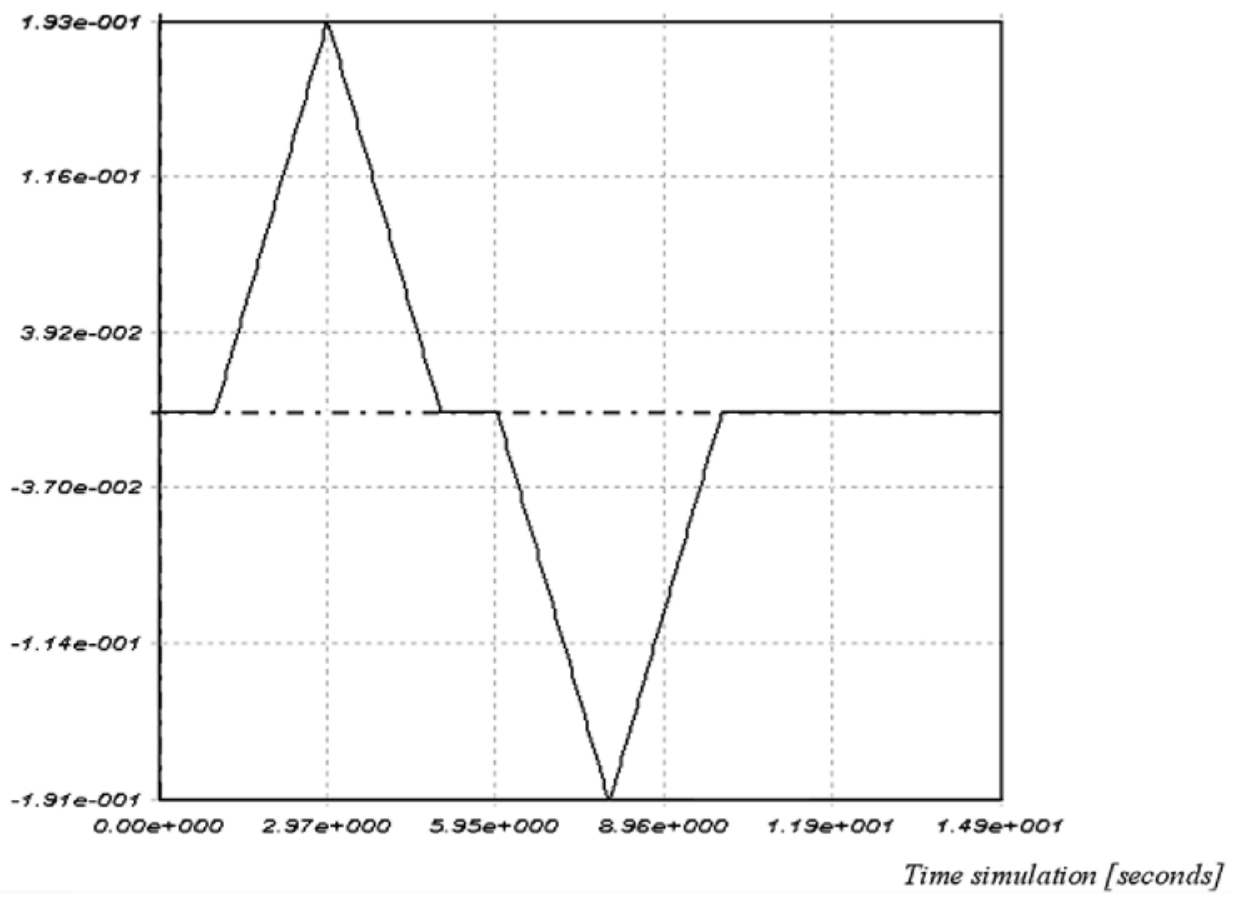

Fig. 14. Movement of the hydraulic actuator.

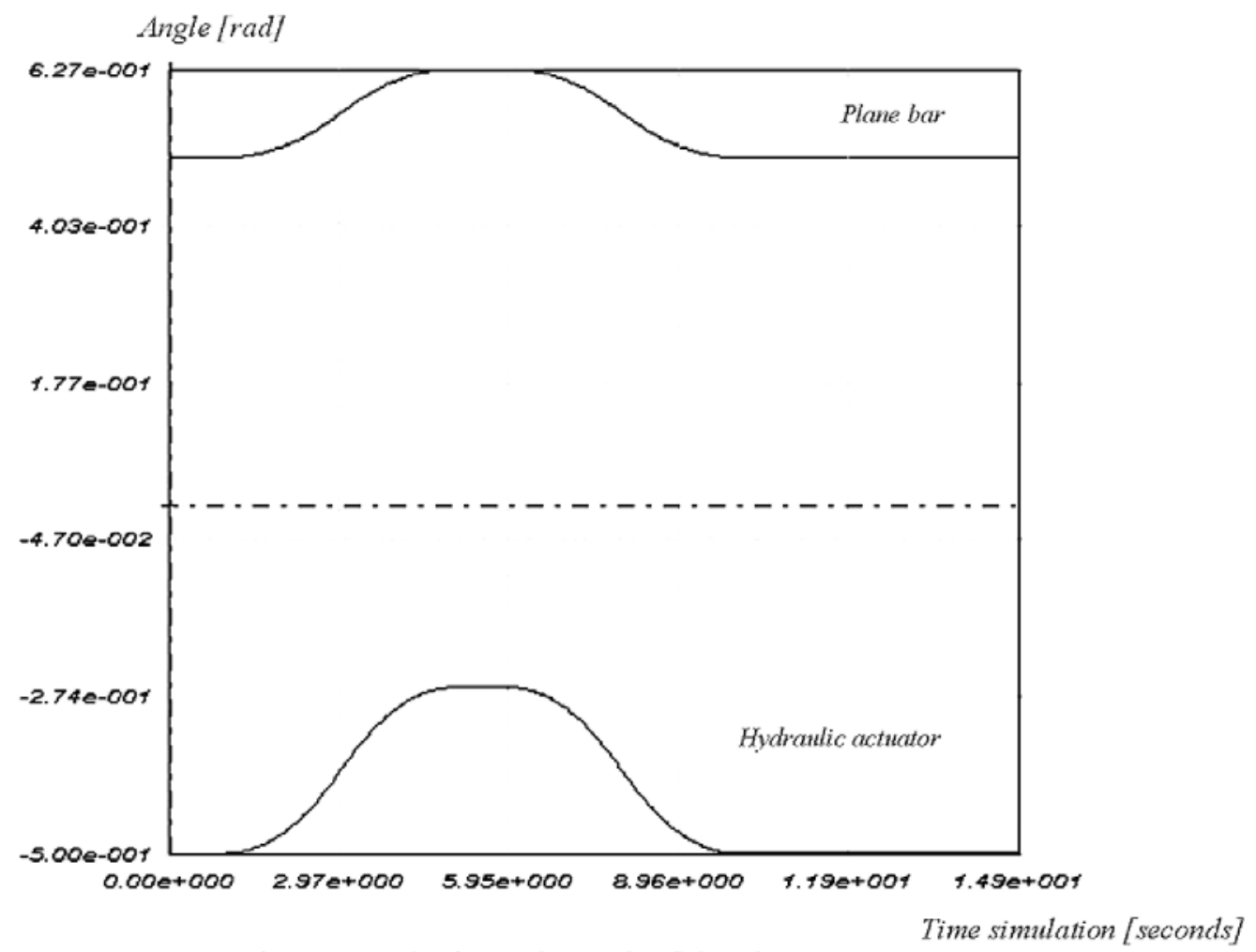

Fig. 15. Angle drawn by each of the elements. 
The relevance of the inserted spring to take account of the displacement of the hydraulic actuator (Kr) may also be seen, which is simply to transform the speed into a displacement so as to be able to subsequently change the length of the hydraulic actuator.

$$
\begin{aligned}
& \frac{\mathrm{d}}{\mathrm{d} t} X K r(t)=V_{\text {piston }}
\end{aligned}
$$

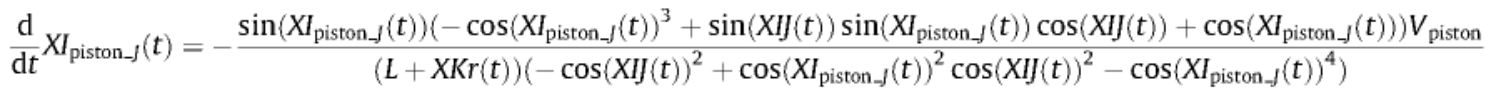

$$
\begin{aligned}
& \frac{\mathrm{d}}{\mathrm{d} t} X I J(t)=\frac{V_{\text {piston }}}{L \sin \left(X I_{\text {piston-J }}(t)-X I J(t)\right)}
\end{aligned}
$$

Fig. 15 shows the result of simulating the previous equations taking $L=2 \mathrm{~m}$. To do this, some initial angles of $0.5 \mathrm{rad}$ and $-0.5 \mathrm{rad}$, respectively, of the bar and the actuator were taken into account, and the linear movement of the actuator piston represented in Fig. 14 was introduced.

In these figures, it can be seen how the angles increase from their initial position when the length of the hydraulic actuator is increased and how the same happens to these angles when the hydraulic actuator returns to its initial length.

\section{Plane loading mechanism of a front-loader}

In order to generate the Bond-Graph model for this mechanism, each of the elements of which it is made up will be started from in isolation (Fig. 16), and after generating the Bond-Graph (Fig. 17) layout for each of them, they will be grouped and assembled.

While all the body parts can only be represented by the anchorage points with other elements, in the bucket (part 6), the point located at the end will need to be inserted. On the one hand, this point will serve to insert the reactions produced by interaction with the terrain and due to the weight of the load, and on the other to validate the bucket movement.

In order to facilitate the assembly of all the elements once a Bond-Graph of one element has been made, their complete ports and nodes should be grouped and their entrances and exits left free so that the element can be linked to the rest of the subsets. Once the different subsets of the mechanism have been obtained, it is essential to impose joints on the different nodes using the restrictions of type 1 junction (Fig. 18), finally obtaining the mechanism we are dealing with.

In this way, the number of degrees of freedom will be proportional to the number of rods $(6 \times 3)$ and the restrictions due to joints $(2 \times 8)$. Thus, it can be shown that there are only two degrees of freedom $(6 \times 3-2 \times 8)$, as many as actuators.

After obtaining the equations corresponding to the model, the next step is to validate it by using its numeric simulation.

\section{Validation}

In the type of machines dealt with in this paper, the driving of the machine can be clearly separated from any handling of the implements that operate the bucket, since they must not be done simultaneously in order to avoid the machine overturning, mainly when the bucket is loaded with the material. Generally speaking, the most important reactions occurring on the chassis of the machine are those due to displacement of the load in the bucket (around $5000 \mathrm{~kg}$ ), since this is the only mass that varies, either through volume or density, and which the driver needs to be used to handling.

However, the maximum angle that the push arm can form in respect of the horizontal, where its centre of mass is as far from the machine's centre of gravity as possible and thus where the torque due to the weight of the arm is greater, is $40^{\circ}$. If the maximum front angle is considered, the distances between the machine's centres of gravity and the push arm can fluctuate around $10 \%$. On the other hand, the low speed at which the implements operate (times of 5-6s to raise a full bucket and 2-3 s to lower it when emptying it, with a $4 \mathrm{~m}$. trajectory) and its weights (around $600 \mathrm{~kg}$ per bucket and the push arm $400 \mathrm{~kg}$ ) cause the forces appearing on them to be less significant than those originated by the load movement $(5000 \mathrm{~kg})$.

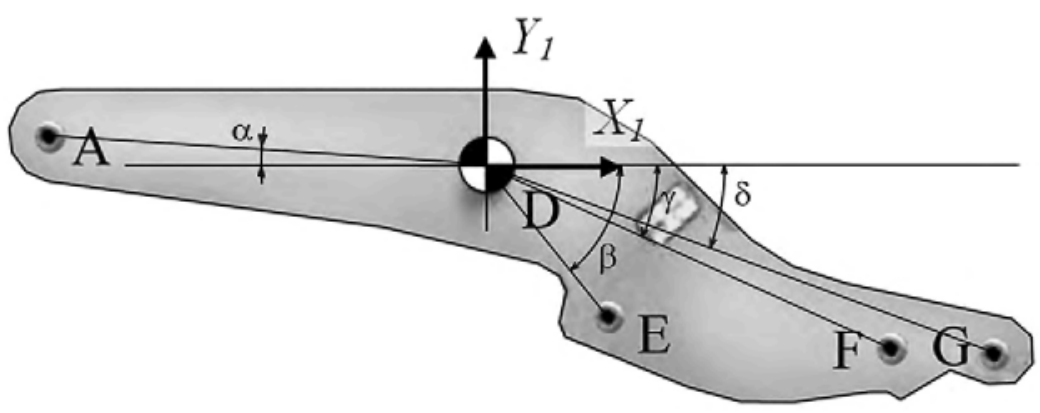

Fig. 16. Support $\operatorname{arm}\left(\right.$ element $n^{\circ}$ 1). 


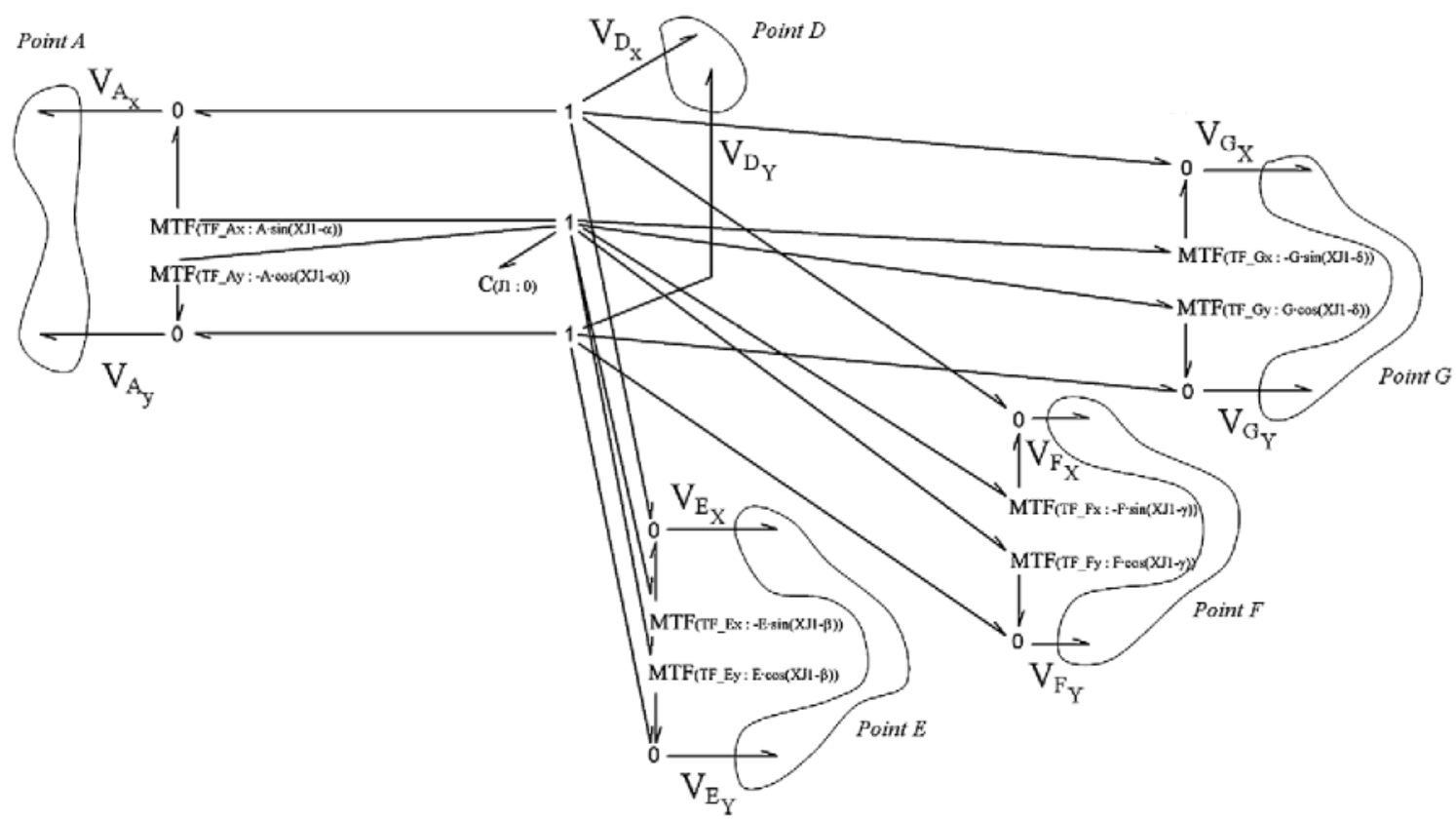

Fig. 17. Support arm (element $n^{\circ} 1$ ) using Bond-Graph.

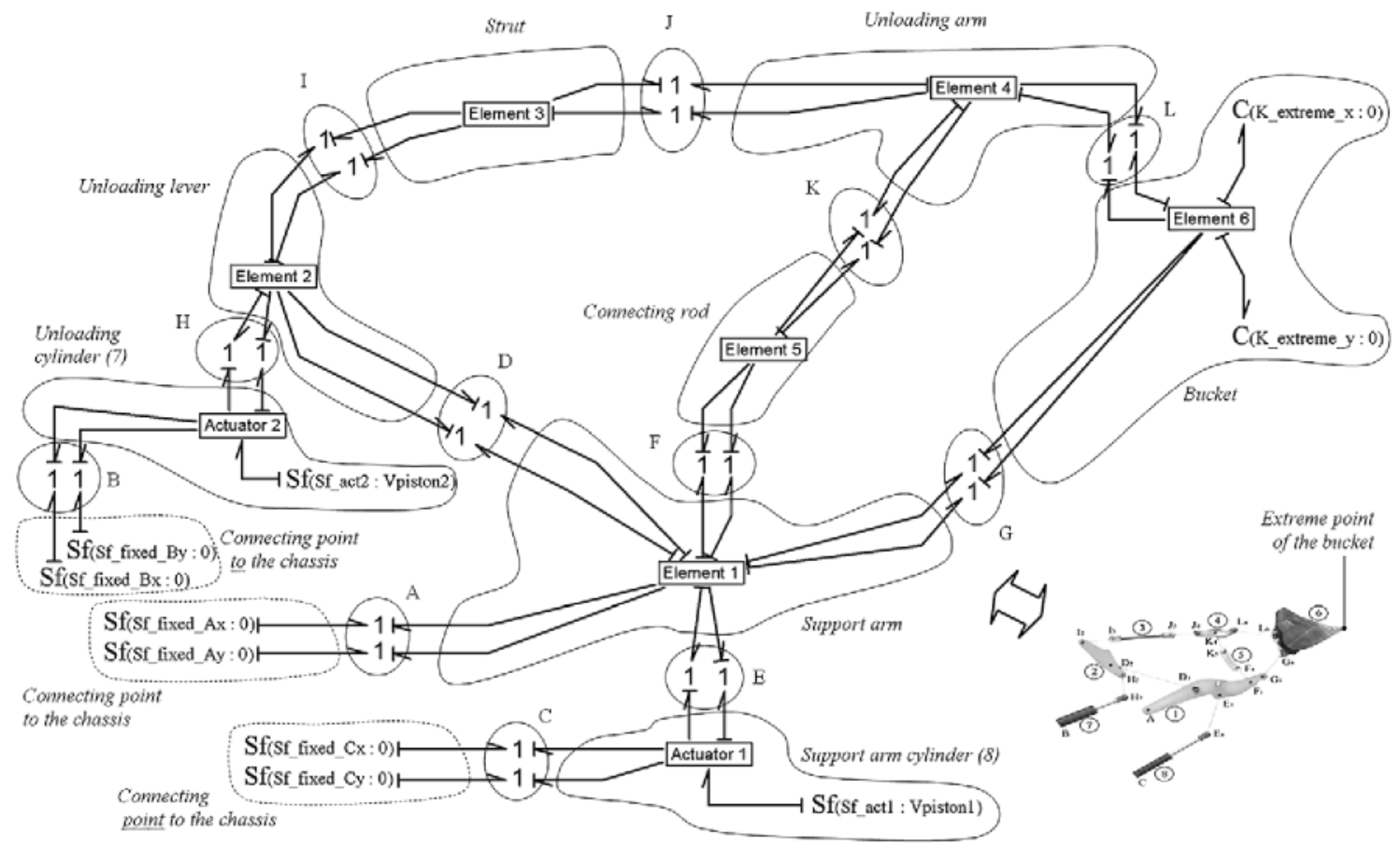

Fig. 18. Front-loader mechanism using a Bond-Graph.

The load on the bucket will not only be affected by the effect of gravity but also by that of the acceleration of the machine and the effects due to inserting the bucket into the material to be loaded, since penetration and material cutting forces are produced. In all cases, these forces that have been mentioned, instead of fastening the planar mechanism to some points located on the three-dimensional solid corresponding to the vehicle and so work with a single model where the reactions themselves would act directly on the chassis, are preferable to isolate the mechanism. 


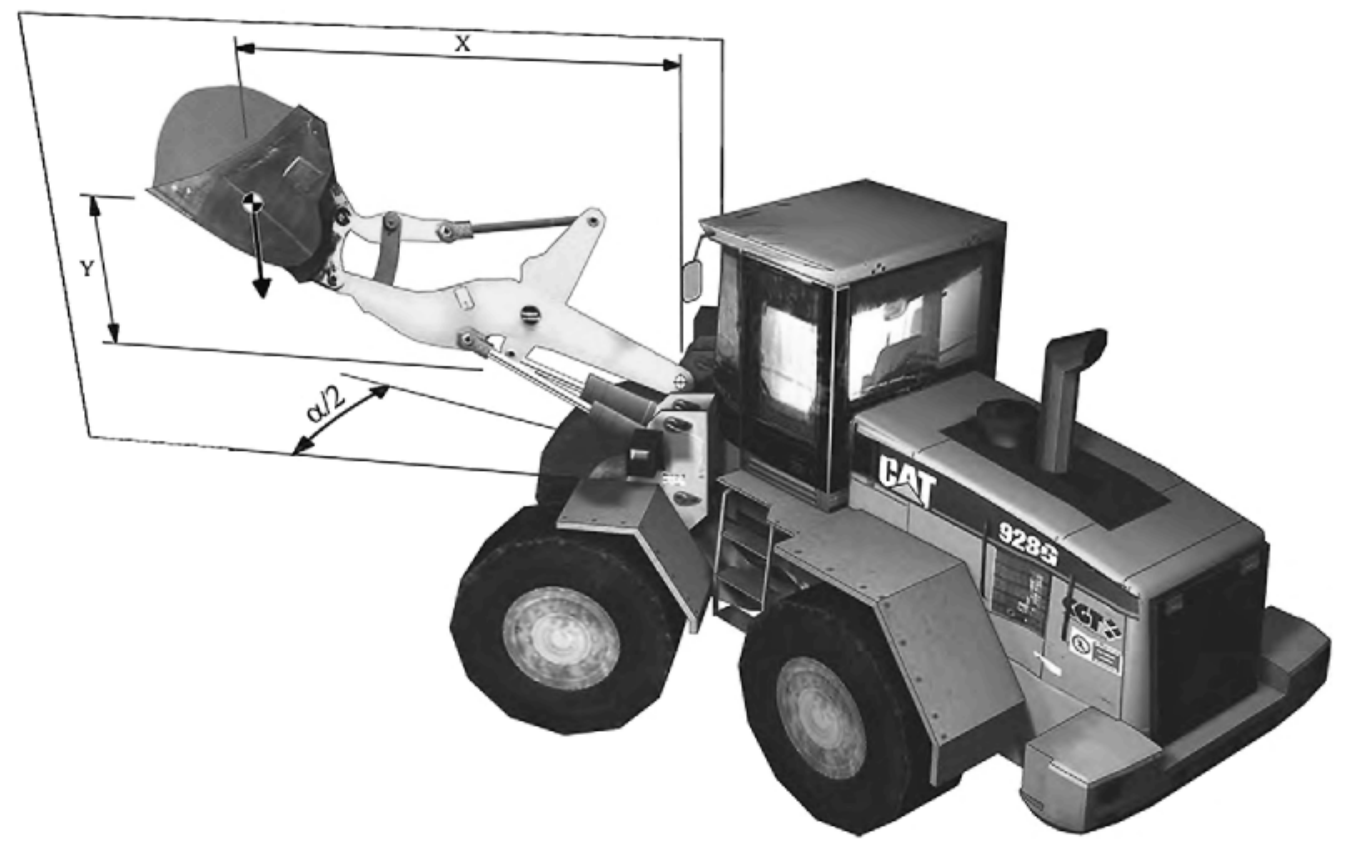

Fig. 19. Effect of the weight load over the front-loader machine.

Therefore, once the kinematic simulation of the articulated arm has been obtained, not only can the angles on each of the arms be obtained, but also the position of the point where the load or the different forces are acting and in this way can directly calculate the torque on the machine chassis due to the load, inertia, the reactions with the terrain and the orientation of the chassis in respect of the implements ( $\alpha / 2$ in following figure). Likewise, it is possible to apply the effect of the machine's acceleration and the mass of each of the implements.

Thus, in order to obtain the performance due to the different reactions on the chassis, all that is needed is to introduce some forces into the three-dimensional solid and some points of application (Fig. 19).

According to the front-loader mechanism using Bond-Graph (Fig. 18), we can start from rest and analyse if the control of the different actuators generates a good movement of the bucket; then, it would be necessary to apply the different effects of the load over the chassis but it is not an objective of this paper.

For these reasons, in simulations aimed at using and handling theses types of machines it is possible to ignore the variation in the torques existing on the chassis due to the masses of the arm implements and only take into account the torque due to load.

The bucket movement consists of the alternative displacement of the two main arms, the support arm and the unloading arm, and it is controlled by the length of the different hydraulic actuators Lcil_1 and Lcil_2. Fig. 20 shows the different phases comprising the bucket movement.

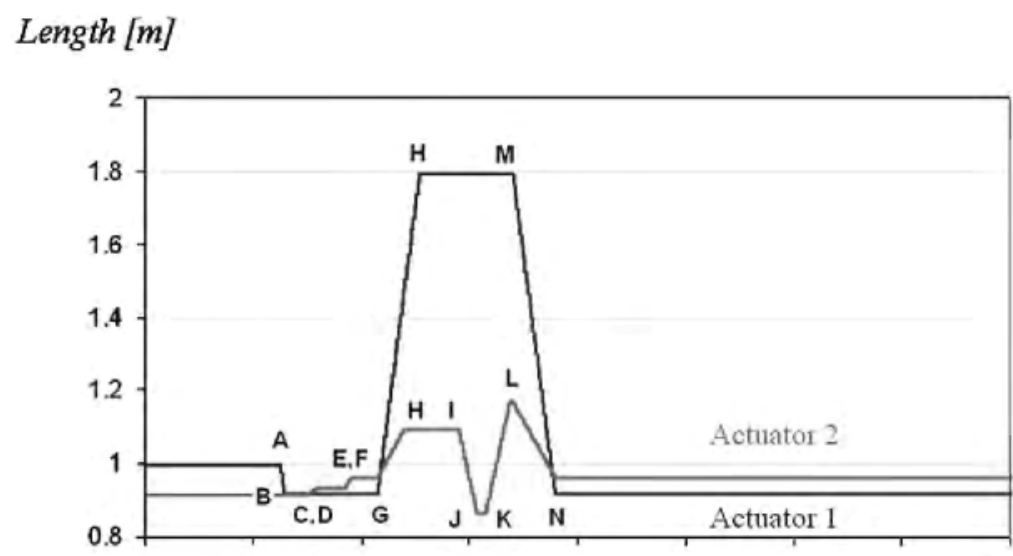

Time simulation [\%]

Fig. 20. Length of the actuators according to time. 


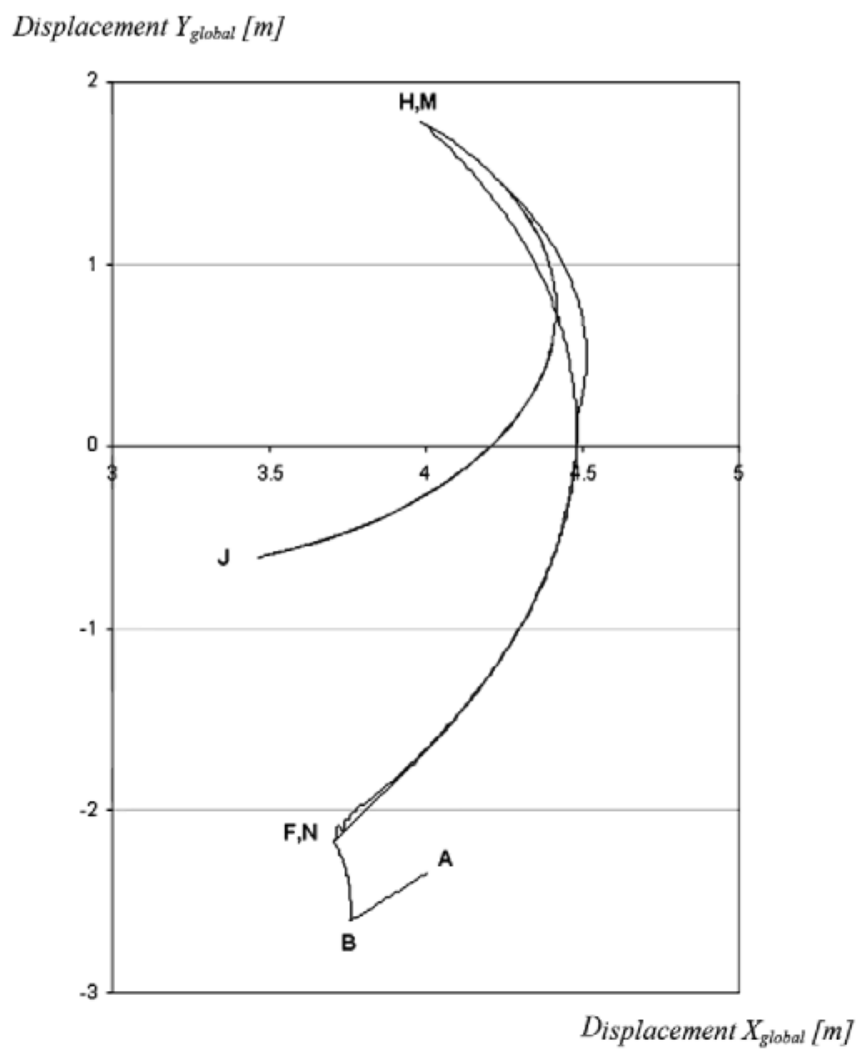

Fig. 21. Path drawn by the end of the bucket

Firstly, starting out from the rest, the support arm actuator is retracted initially in order to approach the bucket to the ground (A-B) and then this is placed in a horizontal position (C-D) by means of the other actuator so that loading can be properly performed (D-E). Once the material has been loaded, it is tilted backwards (E-F) using the unloading cylinder so that the machine can move with the bucket full without spilling the load (F-G). When the place for unloading has been reached, firstly the arm and the load are raised together $(\mathrm{G}-\mathrm{H})$ by means of the two actuators, and secondly it is tilted forward (I-J) with the unloading actuator to empty the contents. Finally, the bucket is tilted backwards (K-L) by means of the unloading actuator and the thrust arm is lowered $(\mathrm{M}-\mathrm{N})$ using the support arm actuator in order to return to the starting position.

To analyse if the length of the different actuators generates the real movement of the bucket, we have selected a representative point as the end of the bucket, represented by the two zero compliances $C_{-}$extreme_$x$ and $C_{-}$extreme_ $y$ shown in Fig. 18. The bucket is initially located horizontally at a short distance from the ground, so as to be able to pick up the load and then raise it and tilt it to unload the material.

Since this is a mechanism for moving the bucket, the movement described by the path drawn by the point located at the end of the bucket during its simulation is shown in Fig. 21.

Fig. 21 shows how the end descends from the initial position (A) to a point located lower down (B), then it is tilted (F), partly to place the bucket horizontally and partly to hold firm the loaded material. Then, it is raised to unload the bucket $(\mathrm{H})$ and afterwards, after tilting it, it moves to a point located lower down $(\mathrm{J})$. Once the material has been unloaded, the bucket returns to its position near to its original position after tilting $(\mathrm{M})$ and descent $(\mathrm{N})$.

\section{Simulation time analysis}

A real time simulator has to graphically represent the behaviour of the entire machine at least 30 times per second in order to obtain a realistic effect. Fewer times would mean that the human eye would perceive something strange and not associate what is being simulated with something real. However, to visually represent the different parts of the simulation environment, the console inside the cab, etc., and to be able to reproduce the movements on a platform of several degrees of freedom, a certain computation time has to be used on the one hand, and on the other a certain communication time between the different computers has to be used. 


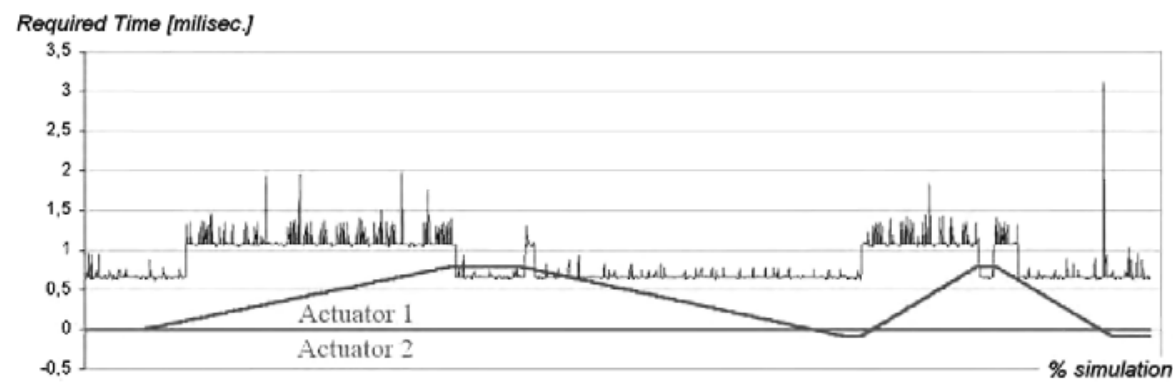

Fig. 22. Times employed in solving the arm-bucket kinematics.

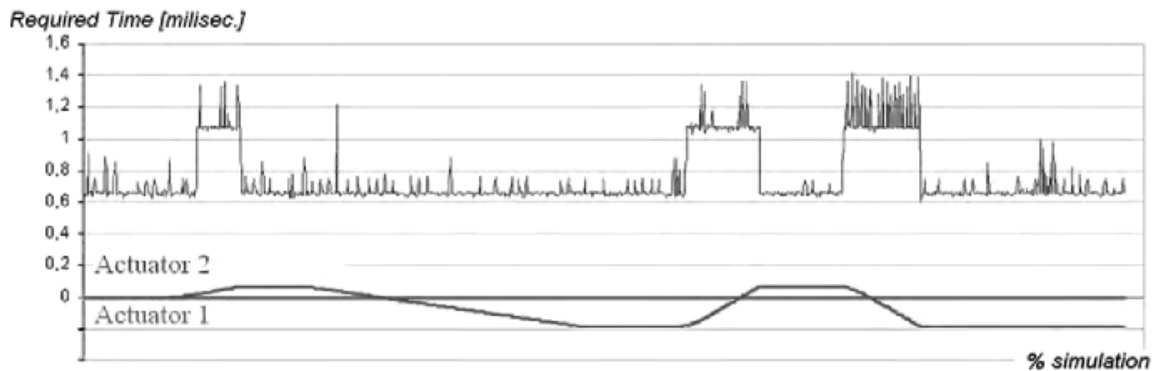

Fig. 23. Times employed in solving the arm-bucket kinematics.

Therefore, the different reactions of the machine with the environment have to be obtained at least 30 times per second. The system of equations of the mathematical model has to be solved and finally it has to be transmitted and represented correctly. To do this properly in the time set, half the time used is usually required to obtain the different reactions of the machine and to solve the system of equations of the mathematical model, and the other half for transmission and representation.

In order to be able to say that the system of equations set out as well as their being solved with the methodology proposed in this paper can be implemented in a real time simulator, under no circumstances should the time exceed $1 / 60 \mathrm{~s}$, that is to say $16.7 \mathrm{~ms}$, including the machine dynamics and the calculation of the penetration forces necessary to move the load.

To perform the time analysis of the times required, the arm-bucket kinematics should be studied, the total time employed being the sum of the times required for it and the machine dynamics. The computer is a Pentium IV, with a $2.4 \mathrm{GHz}$ processor and a $512 \mathrm{Mb}$ RAM memory.

As for the kinematic behaviour of the arm, the behaviour of each hydraulic actuator will be isolated initially and then its action will be studied jointly with the other.

As can be seen in Fig. 22, the time employed fluctuates between 0.7 and $1.5 \mathrm{~ms}$. It can be clearly seen how the maximum times correspond to the handling of the actuator that moves the thrust arm. It can also be seen how the maximum times appear when the arm is in the highest raised position, it being more noticeable when being raised than when being lowered and how operating more quickly or slowly involves no time differences. However, there are some points that reach 2 or 3 ms for no apparent reason.

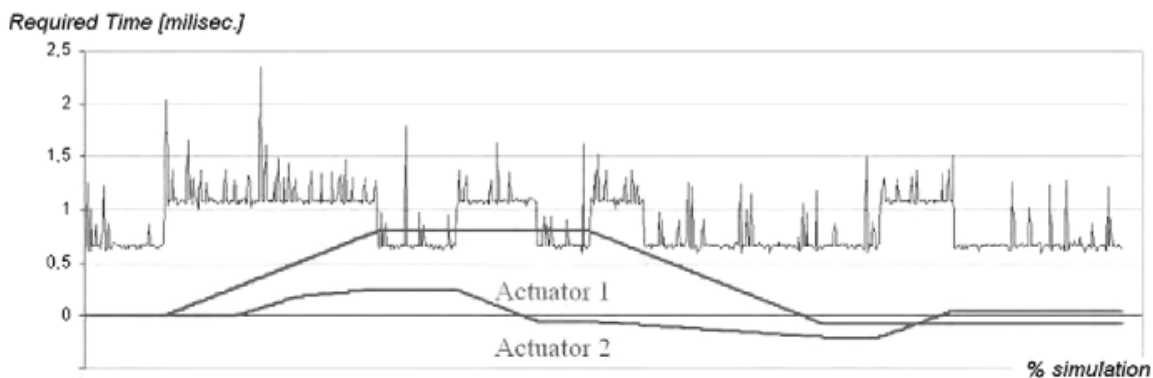

Fig. 24. Times employed in solving the arm-bucket kinematics. 
As for the behaviour of the actuator that tilts the bucket, it can be seen how (Fig. 23), the same as with the other actuator, the maximum times occur when it is being operated. However, contrary to the case of the other actuator, there is definitely a clear difference between operating more quickly or more slowly; the range of long times is greater when operating is quicker. Thus, computation times fluctuate between 0.7 and $1.4 \mathrm{~ms}$.

Finally, as Fig. 24 shows, if the effect of the two actuators is combined, the influence of each one will be seen, with times required fluctuating between 0.7 and $2 \mathrm{~ms}$, with some isolated point reaching $2.4 \mathrm{~ms}$.

If we want to simulate the full machine, it is necessary to add the other controls, the engine, wheels, suspension, chassis, and steering system too and analyse the previous figures corresponding to the simulation times of the mechanism. It is possible to understand why it is necessary to analyse the different parts in order to reduce the time employed in the simulation of each machine part if we want to generate simulations in real time.

\section{Conclusions}

Taking these results as a basis, the advantage of setting out the elements using a Bond-Graph as presented here stems from being able to set out the model, and thus the equations of a kinematic model, according to the displacements and angular velocities instead of the external forces. This makes it possible to find the equations typical of the model, not only in smaller number, but also in less computation time needed to calculate them.

Although it is true that the problems of causality assignment will be the same in most cases, and it is necessary in both cases to assign random causality on intermediate graphs, thereby originating the same number of algebraic equations, in the way it is set out here, no more algebraic equations are added, as no causalities differences exist, and the number of variables and parameters needed is reduced by reducing the number of ports and because these are of zero value.

Finally, when dealing with mechanisms as complex as those of the wheel loader, the use of uncoupling elements is usually resorted to, which makes real time calculation unviable due to stiff behaviour. Should the solution to use stiff joints be adopted, the existence of three inertias with differential causality for each rod and the imposition of causality on intermediate graphs make calculation by DAE or ODE systems unapproachable, meaning that simulation is not possible.

\section{References}

[1] A.M. Bos, Implicit solutions to constraints in mechanical bond graphs, in: Proceedings IMACS Ist World Congress on Systems Simulation and Scientific Computation, Oslo, 1985, vol. 4, pp. 309-312.

[2] D.C. Karnopp, D.L. Margolis, Analysis and simulation of planar mechanism systems using Bond-Graph, J. Mech. Des. 101 (2) (1993) $187-191$.

[3] A.M. Bos, Modeling multibody systems in terms of multibond graphs, Ph.D. thesis, Twente University, Enschede, The Netherlands, 1986.

[4] P.J. Gawthrop, L.S. Smith, Causal augmentation of bond graphs with algebraic loops, J. Franklin Inst. 329 (2) (1992) 291-303.

[5] W. Borutzky, F. Cellier, Tearing in bond graphs with dependent storage elements, in: Proceedings of the CESA'96, 1996, pp. 1113-1119.

[6] W. Borutzky, Representing discontinuities by sinks of fixed causality, in: International Conference on Bond Graph Modeling and Simulation ICBGM'95, 1995, pp. 65-72.

[7] J. Félez et al, Bondyn: a bond graph based simulation program, Trans. ASME. J. Dyn. Syst. Measur. Control 112 (1990) 717-727.

[8] John D. Lamb et al, Equivalences of bond graph junction structures, in: International Conference on Bond Graph Modeling ICBOM'93, Simulation Series, vol. 25, No. 2, SCS Publishing, San Diego, 1993, pp. 79-84.

[9] J. Félez et al, Deriving simulation models from bond graphs with any combination of topological loop classes, in: International Conference on Bond Graph Modeling and Simulation ICBGM'97, Simulation Series, vol. 29, No. 1, SCS Publishing, Phoenix, 1997, pp. 85-93.

[10] J. Félez et al, Deriving simulation models from bond graphs with any combination of topological loop classes, Journal of the Franklin Institute 337 (2000) 579-600.

[11] J. Van Dijk, P. Breedveld, Simulation of system models containing zero-order causal paths - I. Classification of zero-order causal paths, J. Franklin Inst. $328(5 / 6)(1991)$ 959-979.

[12] G. Romero et al, Optimised procedures for obtaining the symbolic equations of a dynamic system by using the Bond-Graph technique, in: International Conference on Bond Graph Modeling and Simulation ICBGM'05, Simulation Series, vol. 37, No. 1, SCS Publishing, New Orleans, 2005 , pp. 51-58.

[13] G. Romero, et al., Kinematic analysis of mechanism by using bond-graph language, in: 20th European Conference on Modelling and Simulation ECMS 2006, Bonn, 2006, pp. 59-65. 